\title{
Decentralized Asynchronous Coded Caching Design and Performance Analysis in Fog Radio Access Networks
}

\author{
Yanxiang Jiang, Senior Member, IEEE, Wenlong Huang, Mehdi Bennis, Senior Member, IEEE, \\ and Fu-Chun Zheng, Senior Member, IEEE
}

\begin{abstract}
In this paper, we investigate the problem of asynchronous coded caching in fog radio access networks (F-RANs). To minimize the fronthaul load, the encoding set collapsing rule and encoding set partition method are proposed to establish the relationship between the coded-multicasting contents for asynchronous and synchronous coded caching. Furthermore, a decentralized asynchronous coded caching scheme is proposed, which provides asynchronous and synchronous transmission methods for different delay requirements. The closed-form expression of the fronthaul load is established for the special case where the number of requests during each time slot is fixed, and the upper and lower bounds of the fronthaul load are given for the general case where the number of requests during each time slot is random. The simulation results show that our proposed scheme can create considerable coded-multicasting opportunities in asynchronous request scenarios.
\end{abstract}

Index Terms-Fog radio access networks, asynchronous coded caching, coded-multicasting, fronthaul load.

\section{INTRODUCTION}

With the rapid proliferation of smart devices and mobile application services, wireless networks have been suffering an unprecedented data traffic pressure in recent years, especially

Manuscript received May 25, 2018, revised December 2, 2018, and January 13, 2019.

This work was supported in part by the Natural Science Foundation of Jiangsu Province under grant BK20181264, the Research Fund of the State Key Laboratory of Integrated Services Networks (Xidian University) under grant ISN19-10, the Research Fund of the Key Laboratory of Wireless Sensor Network \& Communication (Shanghai Institute of Microsystem and Information Technology, Chinese Academy of Sciences) under grant 2017002, the National Basic Research Program of China (973 Program) under grant 2012CB316004, a Shenzhen Municipality/HITSZ Start-Up Grant entitled "Energy-Efficient Low-Latency Wireless Networks", and the U.K. Engineering and Physical Sciences Research Council under Grant EP/K040685/2. Part of this work has been presented at 2018 IEEE 88th Vehicular Technology Conference (VTC2018-Fall), Chicago, USA, August 2018.

Y. Jiang is with the National Mobile Communications Research Laboratory, Southeast University, Nanjing 210096, China, the State Key Laboratory of Integrated Services Networks, Xidian University, Xi'an 710071, China, and the Key Laboratory of Wireless Sensor Network \& Communication, Shanghai Institute of Microsystem and Information Technology, Chinese Academy of Sciences, 865 Changning Road, Shanghai 200050, China (e-mail: yxjiang@seu.edu.cn).

W. Huang is with the National Mobile Communications Research Laboratory, Southeast University, Nanjing 210096, China (e-mail: ahhw10514@163.com).

M. Bennis is with the Centre for Wireless Communications, University of Oulu, Oulu 90014, Finland (e-mail: mehdi.bennis@oulu.fi).

F. Zheng is with the School of Electronic and Information Engineering, Harbin Institute of Technology, Shenzhen 518055, China, and the National Mobile Communications Research Laboratory, Southeast University, Nanjing 210096, China. (e-mail: fzheng@ieee.org). at peak-traffic moments. Fog radio access networks (F-RANs), which can effectively reduce the data traffic pressure by placing popular contents closer to users, have been receiving significant attention from both industry and academia [1], [2]. In F-RANs, fog access points (F-APs) are distributed at the edges and connected to the cloud server through fronthaul links. F-APs can use edge computing and caching resources to provide users better quality of experience [3]. Meanwhile, since a few popular content resources account for most of the traffic load, edge caching has become instrumental in content delivery [4]-[6]. Moreover, coded caching was firstly proposed in [7] and [8] by encoding the delivered contents to further reduce the data traffic pressure.

The main idea of coded caching is that the contents stored in the caches can be used to create coded-multicasting opportunities, such that a single coded-multicasting content transmitted by the cloud server can be useful to a large number of users simultaneously even though they are not requesting the same content. In [7], Maddah-Ali and Niesen proposed a centralized coded caching scheme, in which the centrally coordinated placement phase needs the knowledge of the number of active users in the delivery phase. A decentralized coded caching scheme was further proposed in [8], which achieves order-optimal memory-load tradeoff in the asymptotic regime with infinite file size. However, when the file size is sub-exponential with respect to the number of users, the Maddah-Ali-Niesen's decentralized scheme in [8] achieves at most a multiplicative gain of two over the conventional uncoded caching scheme [9]. Focusing on the finite file size regime, the authors in [10] proposed a decentralized random coded caching scheme and a partially decentralized sequential coded caching scheme, which outperform the Maddah-AliNiesen's decentralized scheme when the file size is not very large.

Furthermore, the authors in [11] presented a strategy which partitions the file library into subsets of approximately uniform request probability and applies the strategy in [8] to each subset. In [12], the authors considered the case where the entire content is divided into multiple different levels based on popularity and an information-theoretic outer bound was developed. A scheme consisting of a random popularity-based caching policy and chromatic-number index coding delivery was proposed in [13], which was proven to be order optimal in terms of average rate. In [14], the authors considered an arbitrary popularity distribution and derived a new information- 
theoretical lower bound on the expected transmission rate of any coded caching schemes. In [15], the analysis in [13] was extended to the case where each user requests multiple files and an order-optimal delivery scheme was provided based on local graph coloring, which achieved the order gain compared with the repeated application of the scheme in [7]. In [16], the analysis was further extended to the case with distinct cache sizes and demand distributions, and a novel polynomial-time algorithm based on greedy local graph coloring was proposed, which can recover a significant part of the multiplicative caching gain with the same content packetization. In [17] and [18], more complex heterogeneous network settings for file sizes and cache capacities were studied, respectively. In [19], an online coded caching scheme was proposed, which updates cache contents by evicting some old file parts and replacing them with randomly chosen parts of the newly delivered files. In [20], the authors reformulated the centralized coded caching problem as designing a corresponding placement delivery array and proposed two new schemes from this perspective which can significantly reduce the rate compared with the uncoded caching schemes. In [21], the authors viewed the centralized coded caching problem in a hypergraph perspective and showed that designing a feasible placement delivery array is equivalent to constructing a linear hypergraph in extreme graph theory. In [22], the authors proposed coded caching schemes based on combinatorial structures called resolvable designs, which can be obtained in a natural manner from linear block codes whose generator matrices possess certain rank properties, and obtained several schemes with subpacketization levels substantially lower than the basic scheme at the cost of an increased rate. In [23], improved lower bounds on the required rate for the coded caching problem was developed and it was demonstrated that the computation of this lower bound can be posed as a combinatorial labeling problem on a directed tree. In [24], a coded prefetching and the corresponding delivery strategy was proposed, which relies on a combination of rank metric codes and maximum distance separable (MDS) codes in a non-binary finite field. In [25], a novel centralized coded caching scheme was proposed that approaches the ratememory region achieved by the scheme in [24] as the number of users in the system increases, which only requires a finite field of $2^{2}$. Moreover, instead of relying on the existence of some valid code, an explicit combinatorial construction of the caching scheme was provided. In [26], the authors proposed a connection between the uncoded prefetching scheme proposed by Maddah Ali and Niesen and the coded prefetching scheme in [24]. The new general coding scheme was then presented and analyzed rigorously, which yields a new inner bound to the memory-rate tradeoff for the caching problem.

All the above schemes in [7]-[26] considered the coded caching problem for the case in which user requests are synchronous, i.e., synchronous coded caching. However, user requests for contents are typically asynchronous in reality [27]. The asynchronous request case was first mentioned in [8], and the authors applied their proposed decentralized synchronous coded caching scheme to an asynchronous request scenario in a simple way. In [28], the delay sensitive coded caching problem was first studied and the situation whereby each asynchronous request has a specific deadline was considered. Then, a computationally efficient caching scheme that exploits coded-multicasting opportunities was developed subject to the delivery-delay constraint. In [29], the authors proposed a linear programming formulation for the offline case that the server knows the arrival time before starting transmission. As for the online case that user requests are revealed to the server over time, they considered the situation that users do not have deadlines but wish to minimize the overall completion time. In [30], a centralized coded joint pushing and caching (CJPC) method with asynchronous user requests was proposed to minimize the network traffic by jointly determining when and which data packets are to be pushed and whether they should be cached. Optimal offline and online C-JPC policies for noncausal and causal request delay information were obtained by solving optimization problems. Fountain coded caching (FCC) and generalized coded caching (GCC) methods were further proposed to give sub-optimal policies with low complexity. The authors analyzed the bounds on the optimal traffic volume and proved that the FCC and GCC methods achieve optimal or near-optimal traffic volumes in some special cases.

Motivated by the aforementioned discussions, it is important to study the coded caching problem when user requests are asynchronous, i.e., asynchronous coded caching. In view of this, we consider the online case with a given maximum request delay to reduce the worst-case load of the fronthaul links in F-RANs. Our main contributions are summarized below.

1) We propose an encoding set collapsing rule to establish the relationship between the coded-multicasting contents in asynchronous and synchronous coded caching. Furthermore, we propose an encoding set partition method, which can create considerable coded-multicasting opportunities while the delay of each user is no more than the given maximum request delay.

2) We propose a decentralized asynchronous coded caching scheme, which can exploit the created codedmulticasting opportunities effectively. Our proposed scheme is applicable for various asynchronous request scenarios by providing asynchronous and synchronous transmission methods, which can be chosen according to different delay requirements.

3) We derive the closed-form expression of the fronthaul load for our proposed scheme with the special case and establish the upper and lower bounds of the fronthaul load for our proposed scheme with the general case. We show that the fronthaul load using our proposed scheme is at most a constant factor larger than that of the Maddah-Ali-Niesen's decentralized scheme.

4) We validate our theoretical results by using computer simulations, which show that our proposed scheme can create considerable coded-multicasting opportunities in asynchronous request scenarios, and the maximum request delay can be adjusted flexibly to achieve the loaddelay tradeoff.

The rest of this paper is organized as follows. In Section II, the system model is introduced. Our proposed asynchronous 


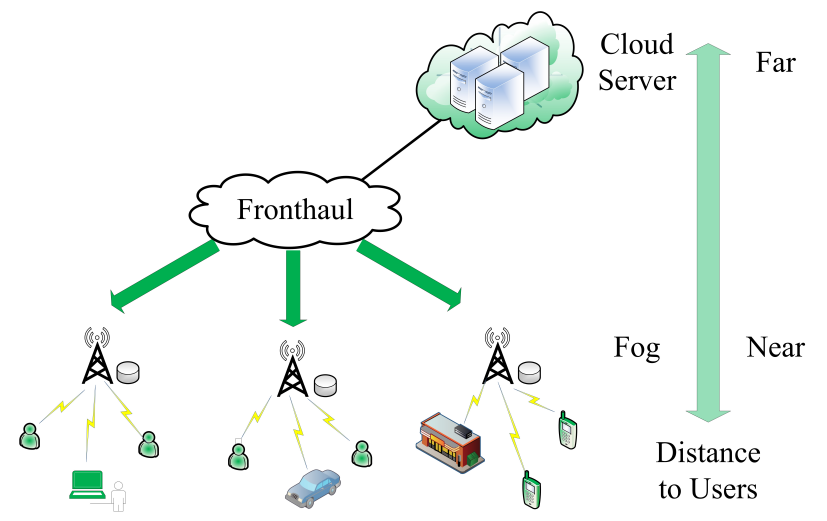

Fig. 1. Illustration of the asynchronous coded caching scenario in the F-RAN.

coded caching scheme is presented in Section III. The performance analysis of our proposed scheme is given in Section IV. In Section V, simulations results are shown. Final conclusions are drawn in Section VI.

\section{SySTEM MODEL}

Consider the F-RAN as shown in Fig. 1 where there are $K$ F-APs and each F-AP serves multiple users. ${ }^{1}$ Assume that the users request contents asynchronously during the time interval $(0, T]$. Let $\mathcal{K}=\{1,2, \ldots, k, \ldots, K\}$ denote the index set of the considered $K$ F-APs. The cloud server has access to a content library of $N$ files, denoted by $W_{1}, W_{2}, \ldots, W_{N}$. Let $\mathcal{N}=$ $\{1,2, \ldots, n, \ldots, N\}$ denote the index set of the $N$ files with $N \geq$ $K$. Assume that the size of each file is $F$ bits and the files in the content library have a uniform popularity distribution. For each F-AP, only one of its served users requests one file during the time interval $(0, T]$, while the F-AP informs the cloud server of the request immediately. For description convenience, we say that $K$ F-APs request contents asynchronously during the considered time interval $(0, T]$, where each F-AP only requests one file. ${ }^{2}$ Each F-AP has an isolated (normalized by $F$ ) cache size $M$ with $0<M<N$.

In the placement phase, the F-APs are given access to the content library. By using the same setting as in [8], F-AP $k$ is able to store its cache content $Z_{k}$ from the content library independently from the other F-APs, i.e., in a decentralized manner. Let $\phi_{k}$ denote the caching function of F-AP $k$, which

\footnotetext{
${ }^{1}$ The network setting here is similar to that in [7]. However, there exist some difference between them. In our network setting, users are connected through F-APs to the cloud server, where an F-AP can store large amount of local data and serve multiple users. Actually, the F-AP can be seen as a sub-server and a request relay station, which can increase the satisfaction of users, improve the efficiency of requests, and reduce the overload of the cloud server.

${ }^{2}$ Multiple requests can also be handled by using our proposed scheme Assume each F-AP can request multiple different files during the considered time interval $(0, T]$. Let $I_{\max }$ denote the maximum allowed number of requested files by one F-AP. Let $d_{k, i}$ denote the index of the $i$ th requested file by F-AP $k$ during $(0, T]$ for $k \in \mathcal{K}$, and $d_{k, i}=\varnothing$ if F-AP $k$ does not request any file for the $i$ th request. Let $D_{1}=\left\{d_{1,1}, d_{2,1}, \ldots, d_{K, 1}\right\}, D_{2}=$ $\left\{d_{1,2}, d_{2,2}, \ldots, d_{K, 2}\right\}, \ldots, D_{I_{\max }}=\left\{d_{1, I_{\max }}, d_{2, I_{\max }}, \ldots, d_{K, I_{\max }}\right\}$. By using our proposed scheme for $D_{1}, D_{2}, \ldots, D_{I_{\max }}$ parallelly and separately, the multiplerequest case can then be handled.
}

maps the content library into the corresponding cache content as follows

$$
Z_{k}=\phi_{k}\left(W_{1}, W_{2}, \ldots, W_{N}\right) .
$$

It can be readily seen that the size of $Z_{k}$ is $M F$ bits.

In the delivery phase, the cache contents of all the F-APs are first communicated to the cloud server, which are then noted as cache records at the cloud server. Without loss of generality, assume that the time interval $(0, T]$ is divided into $B$ time slots with $B \geq 2$. Let $\Delta t=T / B$ denote the time duration of each time slot. Then, time slot $b \in\{1,2, \ldots, B\}$ represents the time interval $((b-1) \Delta t, b \Delta t]$. Let $\mathcal{U}_{b} \subseteq \mathcal{K}$ denote the index set of the F-APs whose requests arrive during time slot $b$ with $\mathcal{U}_{b} \neq \varnothing$. Assume that the cloud server is informed of the requests of the F-APs in $\mathcal{U}_{b}$ during time slot $b$, which are processed in a unified manner, i.e., the cloud server transmits the coded-multicasting content to all the $K$ F-APs through the fronthaul links at the end of each time slot for the online case. Suppose that the maximum request delay that it takes for an F-AP to recover its requested file is $\Delta b \in\{1,2, \ldots, B\}$ time slots. In this paper, we do not consider the time that it takes for the cloud server to transmit the corresponding contents to the F-APs and the time that it takes for each F-AP to transmit the recovered file to the served user. Then, the cloud server should fulfill the requests of the F-APs in $\mathcal{U}_{b}$ by the end of the time slot $b+\Delta b-1$.

Let $d_{k} \in \mathcal{N}$ denote the index of the file requested by F-AP $k$ during $(0, T]$, and $\boldsymbol{d}_{b} \in \mathcal{N}^{\left|\mathcal{U}_{b}\right|}$ denote the request vector of the corresponding F-APs in $\mathcal{U}_{b}$. Let $\psi_{b}$ denote the encoding function of the cloud server at the end of time slot $b$, which maps the files $W_{1}, W_{2}, \ldots, W_{N}$, the cache contents $Z_{1}, Z_{2}, \ldots, Z_{K}$, and the requests $\boldsymbol{d}_{b}$ to the coded-multicasting content as follows

$$
X_{b} \triangleq \psi_{b}\left(W_{1}, W_{2}, \ldots, W_{N}, Z_{1}, Z_{2}, \ldots, Z_{K}, \boldsymbol{d}_{b}\right) .
$$

Let $\theta_{k}$ denote the decoding function of F-AP $k$, which maps the received coded-multicasting contents $X_{b}, X_{b+1}, \ldots, X_{b+\Delta b-1}$, the cache content $Z_{k}$, and the request $d_{k}$ to the estimate of the requested file $W_{d_{k}}$ of F-AP $k$ as follows

$$
\hat{W}_{d_{k}}=\theta_{k}\left(X_{b}, X_{b+1}, \ldots, X_{b+\Delta b-1}, Z_{k}, d_{k}\right) .
$$

Each F-AP should be able to recover its requested file successfully from its cached content and the received codedmulticasting contents, and then transmit it to the served user. For every large enough file size $F$, an asynchronous coded caching scheme is feasible if and only if the worst-case propability of error over all the possible requests $\boldsymbol{d}_{1}, \boldsymbol{d}_{2}, \ldots, \boldsymbol{d}_{B}$ satisfies the following condition

$$
\max _{\boldsymbol{d}_{1}, \boldsymbol{d}_{2}, \ldots, \boldsymbol{d}_{B}} \max _{k \in \mathcal{K}} P\left(\hat{W}_{d_{k}} \neq W_{d_{k}}\right)<\varepsilon, \quad \varepsilon>0 .
$$

The objective of this paper is to find a feasible asynchronous coded caching scheme to minimize the worst-case normalized fronthaul load (over all the possible requests $\boldsymbol{d}_{1}, \boldsymbol{d}_{2}, \ldots, \boldsymbol{d}_{B}$ ) in the delivery phase for a given maximum request delay [31]. 


\section{Proposed Decentralized Asynchronous Coded CACHING SCHEME}

In this section, we first introduce the encoding set collapsing rule. Then, we show the encoding set partition method. Finally, we present the proposed decentralized asynchronous coded caching scheme.

\section{A. The Proposed Encoding Set Collapsing Rule}

Asynchronous coded caching and synchronous coded caching are thought to be under the same condition when their system parameters $M, K$, and $N$ are the same. As the conventional synchronous coded caching scheme under the same condition, such as the Maddah-Ali-Niesen's decentralized scheme, $\mathcal{S} \subseteq \mathcal{K}$ for any $s=|\mathcal{S}| \in \mathcal{K}$ is called an encoding set if a single coded-multicasting content can be useful to the F-APs in $\mathcal{S}$ simultaneously. It can be readily seen that the subset of $\mathcal{S}$ is also an encoding set. In order to differentiate the same subfile in asynchronous and synchronous coded caching, let $W_{k, S}^{\mathrm{a}}$ and $W_{k, S}^{\mathrm{s}}$ denote the bits of the file requested by F-AP $k$ cached exclusively at the F-APs in $\mathcal{S}$ for asynchronous and synchronous coded caching, respectively.

Consider that the requests of the F-APs in $\mathcal{K} \backslash \mathcal{U}_{1}$ have not arrived yet during time slot 1 . Assume $\mathcal{U}_{1} \cap \mathcal{S} \neq \varnothing$ and the requests in $\boldsymbol{d}_{1}$ should be fulfilled at the end of time slot 1. The cloud server needs to transmit a coded-multicasting content which is useful to the F-APs in $\mathcal{U}_{1} \cap \mathcal{S}$ at the end of time slot 1 . Thus, we say that the encoding set $\mathcal{S}$ in synchronous coded caching collapses into a subset of $\mathcal{S}$, i.e., $\mathcal{U}_{1} \cap \mathcal{S}$, for transmitting the corresponding coded-multicasting content in asynchronous coded caching. Recall that by applying the scheme in [8], the coded-multicasting content that the cloud server transmits for $\mathcal{S}$ in synchronous coded caching is $\oplus_{k \in \mathcal{S}} W_{k, \mathcal{S} \backslash\{k\}}^{\mathrm{s}}$, where $\oplus$ denotes the bitwise XOR operation. Accordingly, the cloud server transmits $\oplus_{k \in\left(\mathcal{S} \cap \mathcal{U}_{1}\right)} W_{k,\left(\mathcal{S} \cap \mathcal{U}_{1}\right) \backslash\{k\}}^{\mathrm{a}}$ at the end of time slot 1 . According to the above discussions, it is obvious that there exists some relationship, called encoding set collapsing rule, between the coded-multicasting contents in asynchronous and synchronous coded caching.

During each time slot, let $\mathcal{U}^{\mathrm{y}}$ denote the index set of the F-APs from which the requests have arrived and $\mathcal{U}^{\mathrm{n}}=\mathcal{K} \backslash \mathcal{U}^{\mathrm{y}}$. For any $\mathcal{S}^{1} \subseteq \mathcal{U}^{\mathrm{y}}$ and $\mathcal{S}^{2} \subseteq \mathcal{U}^{\mathrm{n}}$, the encoding set $\mathcal{S}=\mathcal{S}^{1} \cup \mathcal{S}^{2}$ in synchronous coded caching collapses into $\mathcal{S}^{1}$ in asynchronous coded caching. Accordingly, $\oplus_{k \in\left(\mathcal{S}^{1} \cup \mathcal{S}^{2}\right)} W_{k,\left(\mathcal{S}^{1} \cup \mathcal{S}^{2}\right) \backslash\langle k\rceil}^{\mathrm{s}}$ collapses into $\oplus_{k \in \mathcal{S}^{1}} W_{k,\left(\mathcal{S}^{1} \cup \mathcal{S}^{2}\right) \backslash\{k\}}^{\mathrm{a}}$, which will be practically transmitted by the cloud server at the end of the current time slot in asynchronous coded caching.

Example 1: Assume that $N=4, K=4, M=2, B=4$, $T=4 \mathrm{~s}, \Delta t=1 \mathrm{~s}, \Delta b=2, \mathcal{U}_{b}=\{b\}$ and $d_{k}=k$. Consider the encoding set $\{1,2,3,4\}$, and the corresponding coded-multicasting content in synchronous coded caching is $W_{1,\{2,3,4\}}^{\mathrm{s}} \oplus W_{2,\{1,3,4\}}^{\mathrm{s}} \oplus W_{3,\{1,2,4\}}^{\mathrm{s}} \oplus W_{4,\{1,2,3\}}^{\mathrm{s}}$. Note that the request of F-AP 1 should be fulfilled by the end of time slot 2 . $W_{3,\{1,2,4\}}^{\mathrm{s}}$ and $W_{4,\{1,2,3\}}^{\mathrm{s}}$ cannot be encoded together at the end of time slot 2 because the requests of F-AP 3 and F-AP 4 have not arrived yet. Correspondingly, $W_{1,\{2,3,4\}}^{\mathrm{s}} \oplus W_{2,\{1,3,4\}}^{\mathrm{s}} \oplus W_{3,\{1,2,4\}}^{\mathrm{s}} \oplus W_{4,\{1,2,3\}}^{\mathrm{s}}$ collapses into $W_{1,\{2,3,4\}}^{\mathrm{a}} \oplus W_{2,\{1,3,4\}}^{\mathrm{a}}$, and it will be practically transmitted at the end of time slot 2.

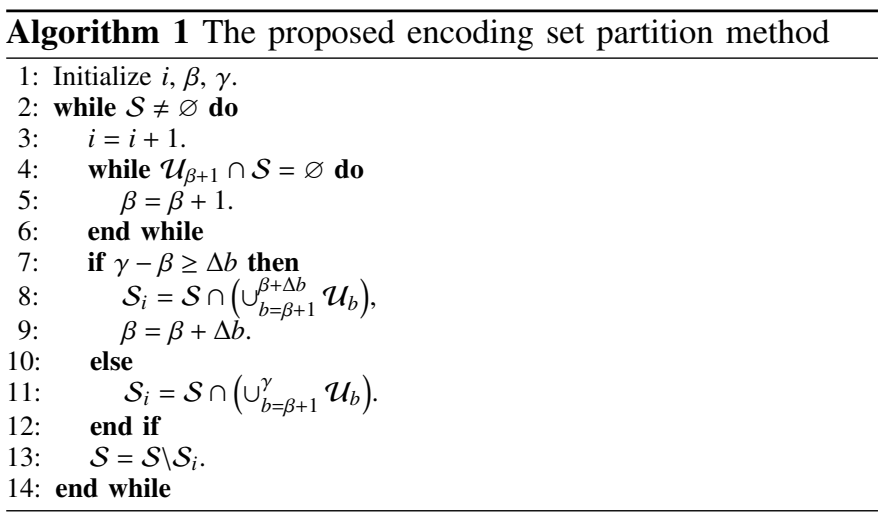

\section{B. The Proposed Encoding Set Partition Method}

Utilizing our proposed encoding set collapsing rule, we now consider what contents are transmitted in asynchronous coded caching. In order to fulfill the requests of the F-APs in asynchronous coded caching, $\mathcal{S}$ may need to be collapsed into a subset of $\mathcal{S}$ many times to transmit the corresponding content at the end of different time slots. For a given $\Delta b$, the requests of the F-APs in $\mathcal{U}_{b-\Delta b+1}$ need to be fulfilled by the end of time slot $b$. Let $\mathcal{U}^{\mathrm{a}}=\cup_{i=\max \{1, b-\Delta b+1\}}^{b} \mathcal{U}_{i}$ denote the index set of the active F-APs during time slot $b$. Then, only the files requested by the F-APs in $\mathcal{U}^{\mathrm{a}}$ can be encoded with each other, which means that $\mathcal{S}$ collapses into $\mathcal{S} \cap \mathcal{U}^{\text {a }}$. Moreover, minimizing the fronthaul load is equivalent to partitioning $\mathcal{S}$ into the minimum number of nonoverlapping subsets for transmission in asynchronous coded caching.

Let $(\beta \Delta t, \gamma \Delta t]$ denote the active time interval of $\mathcal{S}$ if $\left(\left(\cup_{b=1}^{\beta} \mathcal{U}_{b}\right) \cup\left(\cup_{b=\gamma+1}^{B} \mathcal{U}_{b}\right)\right) \cap \mathcal{S}=\varnothing$ and $\mathcal{U}_{b} \cap \mathcal{S} \neq \varnothing$ for $b=\beta+1$ and $b=\gamma$, where $\beta$ and $\gamma$ are integers with $0 \leq \beta<\gamma \leq B$. Suppose that $\mathcal{S}$ is partitioned into $\eta_{\mathcal{S}}(\Delta b)$ subsets for a given $\Delta b$, where $\eta_{\mathcal{S}}(\Delta b)$ is a function of $\Delta b$. Let $\mathcal{S}_{i}$ denote the $i$-th partitioned encoding subset. The detailed encoding set partition method is presented in Algorithm 1.

Example 2: Consider the same setting as Example 1. Focus on $\mathcal{S}=\{1,3,4\}$ with its active time interval $(0,4]$. First, partition $\mathcal{S}$ from time slot 1 , and assign $\mathcal{S} \cap\left(\cup_{b=1}^{2} \mathcal{U}_{b}\right)=\{1\}$ to the first encoding subset $\mathcal{S}_{1}$. Then, let $\mathcal{S}=\mathcal{S} \backslash \mathcal{S}_{1}=\{3,4\}$. Partition $\mathcal{S}$ from the earliest time slot where there is at least one F-AP requesting contents in $\mathcal{S}$, i.e., time slot 3 , and assign $\mathcal{S} \cap\left(\cup_{b=3}^{4} \mathcal{U}_{b}\right)=\{3,4\}$ to the second encoding subset $\mathcal{S}_{2}$. Correspondingly, $\{1,3,4\}$ is partitioned into $\eta_{\mathcal{S}}(2)=2$ encoding subsets, i.e., $\{1\}$ and $\{3,4\}$.

Remark 1: According to Algorithm 1, if $\Delta b_{1}>\Delta b_{2}$, we have

$$
\eta_{\mathcal{S}}\left(\Delta b_{1}\right) \leq \eta_{\mathcal{S}}\left(\Delta b_{2}\right) .
$$

Remark 2: It is possible that there exist some time slots in $(\beta \Delta t, \gamma \Delta t]$, during which no F-APs request contents. Let $B_{\mathcal{S}} \leq B$ denote the number of the time slots in the active time interval $(\beta \Delta t, \gamma \Delta t]$. Then, we have

$$
1 \leq \eta_{\mathcal{S}}(\Delta b) \leq\left\lceil B_{\mathcal{S}} / \Delta b\right\rceil \leq\lceil B / \Delta b\rceil,
$$

where $\lceil\cdot\rceil$ denotes the ceil operation. Specifically, $\eta_{\mathcal{S}}(\Delta b)=$ 1 means that $\mathcal{S}$ will not be partitioned and the cloud server only needs to transmit a single coded-multicasting content, 
that is useful to all the F-APs in $\mathcal{S}$, without increasing extra fronthaul load in asynchronous coded caching. Moreover, it can be readily seen that $\eta_{\mathcal{S}}(\Delta b)=1$ for any $\mathcal{S}$ only when $\Delta b=B$.

\section{The Proposed Asynchronous Coded Caching Scheme}

According to the above discussions, we propose the following decentralized asynchronous coded caching scheme which exploits the encoding set collapsing rule and implements the encoding set partition method. In the placement phase, each F-AP randomly selects $M F / N$ bits of each file with uniform probability and fetch them to fill its cache, which is the same as the Maddah-Ali-Niesen's decentralized synchronous coded caching scheme. Note that the placement procedure does not require any coordination and can be operated in a decentralized manner. More specifically, our proposed scheme can operate in the placement phase with an unknown number of F-APs. In the delivery phase, we propose the following asynchronous and synchronous transmission methods for the online case, which can be chosen by the cloud server. Note that asynchronous or synchronous here means that the cloud server transmits the coded-multicasting contents asynchronously or synchronously.

1) Asynchronous Transmission Method: When $\Delta b<B$, the asynchronous transmission method is chosen. The requests in $\boldsymbol{d}_{b-\Delta b+1}$ need to be fulfilled by the end of time slot $b$ in order that sufficient coded-multicasting opportunities can be created. If $\Delta b>1$, no contents need to be transmitted at the end of time slot $1,2, \ldots, \Delta b-1$, and only the corresponding requests of the active F-APs need to be fulfilled by the cloud server at the end of the time slots between $\Delta b$ and $B$. For description convenience, we say the subfile $W_{k, S}^{\mathrm{a}}$ is of type $s$ with $s=|S|$. Thus, the cloud server transmits a single coded-multicasting content for the F-APs in $\mathcal{S}$ by encoding the subfiles of type $s-1$ [8]. Similarly, we also say the encoding set $\mathcal{S}$ is of type $s$. At the end of the current time slot, the cloud server firstly partitions each file $W_{n}$ into nonoverlapping subfiles [8], whose sizes are calculated according to the updated cache records.

During time slot $b-\Delta b+1$, according to the proposed encoding set collapsing rule in Section III-A, we have: $\mathcal{U}^{\mathrm{y}}=$ $\mathcal{U}_{b-\Delta b+1}$ and $\mathcal{U}^{\mathrm{n}}=\mathcal{K} \backslash \mathcal{U}_{b-\Delta b+1}$. For any $\mathcal{S}^{1} \subseteq \mathcal{U}_{b-\Delta b+1}$ and $\mathcal{S}^{2} \subseteq \mathcal{K} \backslash \mathcal{U}_{b-\Delta b+1}$, the encoding set $\mathcal{S}=\mathcal{S}^{1} \cup \mathcal{S}^{2} \subseteq \mathcal{K}$ for any $s \in \mathcal{K}$ in synchronous coded caching collapses into $\mathcal{S}^{1}$ in asynchronous coded caching. Furthermore, according to the proposed encoding set partition method in Section III$\mathrm{B}$, only the files requested by the active F-APs in $\mathcal{U}^{\mathrm{a}}$ can be encoded with each other, which means that $\mathcal{S}$ collapses into $\mathcal{S} \cap \mathcal{U}^{\text {a }}$, i.e., $\left(\mathcal{S}^{1} \cup \mathcal{S}^{2}\right) \cap \mathcal{U}^{\text {a }}$, by the end of time slot $b$. Let $\chi=\left|\mathcal{S}^{1}\right|$ and $s-\chi=\left|\mathcal{S}^{2}\right|$. Suppose $\chi \in\{\underline{\chi}, \underline{\chi}+1, \ldots, \bar{\chi}\}$, where $\chi$ and $\bar{\chi}$ denote the minimum and maximum of $\chi{ }^{3}$ respectively. By considering any $\chi \in\{\chi, \chi+1, \ldots, \bar{\chi}\}$ with any $s \in \mathcal{K}$, the requests of the F-APs in $\overline{\mathcal{U}}_{b-\Delta b+1}$ can be fulfilled

\footnotetext{
${ }^{3}$ In order that the coded-multicasting content transmitted to the F-APs in $\mathcal{S}$ by the cloud server is useful to at least one F-AP in $\mathcal{U}_{b-\Delta b+1}, \mathcal{S}^{1} \cap \mathcal{S} \neq$ $\varnothing$, i.e., $\chi \geq 1$, needs to be guaranteed. Since $\mathcal{S}^{2} \subseteq \mathcal{K} \backslash \mathcal{U}_{b-\Delta b+1}$, we have $\left|\mathcal{S}^{2}\right|=s-\chi \leq|\mathcal{K}|-\left|\mathcal{U}_{b-\Delta b+1}\right|$. Then, we have $\chi \geq s+\left|\mathcal{U}_{b-\Delta b+1}\right|-|\mathcal{K}|$. Therefore, $\chi=\max \left\{1, s+\left|\mathcal{U}_{b-\Delta b+1}\right|-|\mathcal{K}|\right\}$. Besides, it can be easily verified that $\bar{\chi}=\min \left\{s,\left|\mathcal{U}_{b-\Delta b+1}\right|\right\}$.
}

by the end of time slot $b$ with sufficient coded multicasting opportunities being created for the F-APs in $\left(\mathcal{S}^{1} \cup \mathcal{S}^{2}\right) \cap \mathcal{U}^{\text {a }}$. Recall that the F-APs in $\left(\mathcal{S}^{1} \cup \mathcal{S}^{2}\right) \backslash\{k\}$ share a subfile which is not available in the cache content $Z_{k}$ and requested by $\mathrm{F}$ AP $k \in\left(\mathcal{S}^{1} \cup \mathcal{S}^{2}\right)$. For any $\mathcal{S}^{1}$ and $\mathcal{S}^{2}$ with any $s$, in order to avoid transmitting subfiles repeatedly, no contents need to be transmitted if $W_{k,\left(\mathcal{S}^{1} \cup \mathcal{S}^{2}\right) \backslash\{k\}}^{\mathrm{a}}=\varnothing$ for $k \in\left(\left(\mathcal{S}^{1} \cup \mathcal{S}^{2}\right) \cap \mathcal{U}^{\mathrm{a}}\right)$. Otherwise, the cloud server transmits the coded-multicasting content by the end of time slot $b$ as follows

$$
\oplus_{k \in\left(\left(\mathcal{S}^{1} \cup \mathcal{S}^{2}\right) \cap \mathcal{U}^{\mathrm{a}}\right)} W_{k,\left(\mathcal{S}^{1} \cup \mathcal{S}^{2}\right) \backslash\{k\}}^{\mathrm{a}} \cdot
$$

After the transmission is completed, each F-AP in $\mathcal{U}_{b-\Delta b+1}$ recovers the desirable subfiles of its requested file. Then, each F-AP in $\mathcal{U}_{b-\Delta b+1}$ transmits the recovered subfiles and the corresponding subfiles available in its cache to its served user immediately. Correspondingly, the user can recover the desirable file. Each F-AP in $\mathcal{U}^{\mathrm{a}} \backslash \mathcal{U}_{b-\Delta b+1}$ also recovers the corresponding desirable subfiles and transmits them to its served user at this time. In addition, the cloud server needs to update the cache records of the active F-APs by adding a record of the subfiles recovered by each F-AP in $\mathcal{U}^{\mathrm{a}}$ as its cache content at the end of this time slot. Note that updating the cache records has no influence on the cache contents of the F-APs, which stay unchanged in the delivery phase. The cache records can help the cloud server identify whether the subfile to be transmitted is $\varnothing$ or not in real time before transmission.

At the end of time slot $B$, all the requests of the FAPs in $\mathcal{U}^{\mathrm{a}}$ should be fulfilled together. Similarly, define $\chi^{\prime}=\max \left\{1, s+\left|\mathcal{U}^{\mathrm{a}}\right|-|\mathcal{K}|\right\}$ and $\bar{\chi}^{\prime}=\min \left\{s,\left|\mathcal{U}^{\mathrm{a}}\right|\right\}$. Focus on $\overline{\mathcal{S}}^{1} \subseteq \mathcal{U}^{\mathrm{a}}$ with $\chi=\left|\mathcal{S}^{1}\right|$ and $\mathcal{S}^{2} \subseteq \mathcal{K} \backslash \mathcal{U}^{\mathrm{a}}$ with $s-\chi=\left|\mathcal{S}^{2}\right|$. For any $s \in \mathcal{K}$ and any $\chi \in\left\{\underline{\chi}^{\prime}, \underline{\chi}^{\prime}+1, \ldots, \bar{\chi}^{\prime}\right\}$, the cloud server transmits the coded-multicasting content by the end of time slot $B$ as follows

$$
\bigoplus_{k \in \mathcal{S}^{1}} W_{k,\left(\mathcal{S}^{1} \cup \mathcal{S}^{2}\right) \backslash\{k\}}^{\mathrm{a}},
$$

where all the subfiles $W_{k,\left(\mathcal{S}^{1} \cup \mathcal{S}^{2}\right) \backslash\{k\}}^{\mathrm{a}}$ are assumed to be zeropadded to the number of bits of the longest subfile in the bitwise XOR operation. After that, each F-AP in $\mathcal{U}^{\mathrm{a}}$ recovers the subfiles of its requested file, and then transmits the recovered subfiles and the subfiles available in its cache to its served user. Correspondingly, the user can recover the desirable file.

2) Synchronous Transmission Method: When $\Delta b=B$, the synchronous transmission method is chosen. Firstly, no contents need to be transmitted at the end of time slot $1,2, \ldots, B-1$. At the end of time slot $B$, for all $\mathcal{S}$ with any $s$, the cloud server transmits the coded-multicasting content as follows

$$
\oplus_{k \in \mathcal{S}} W_{k, \mathcal{S} \backslash\{k\}}^{\mathrm{a}} .
$$

Then, each F-AP transmits all the subfiles of its requested file to its served user. Correspondingly, the user can recover the desirable file.

The detailed description of our proposed decentralized asynchronous coded caching scheme is presented in Algorithm 2. Note that the problem setting allows for a vanishing probability of error as $F \rightarrow \infty$. 


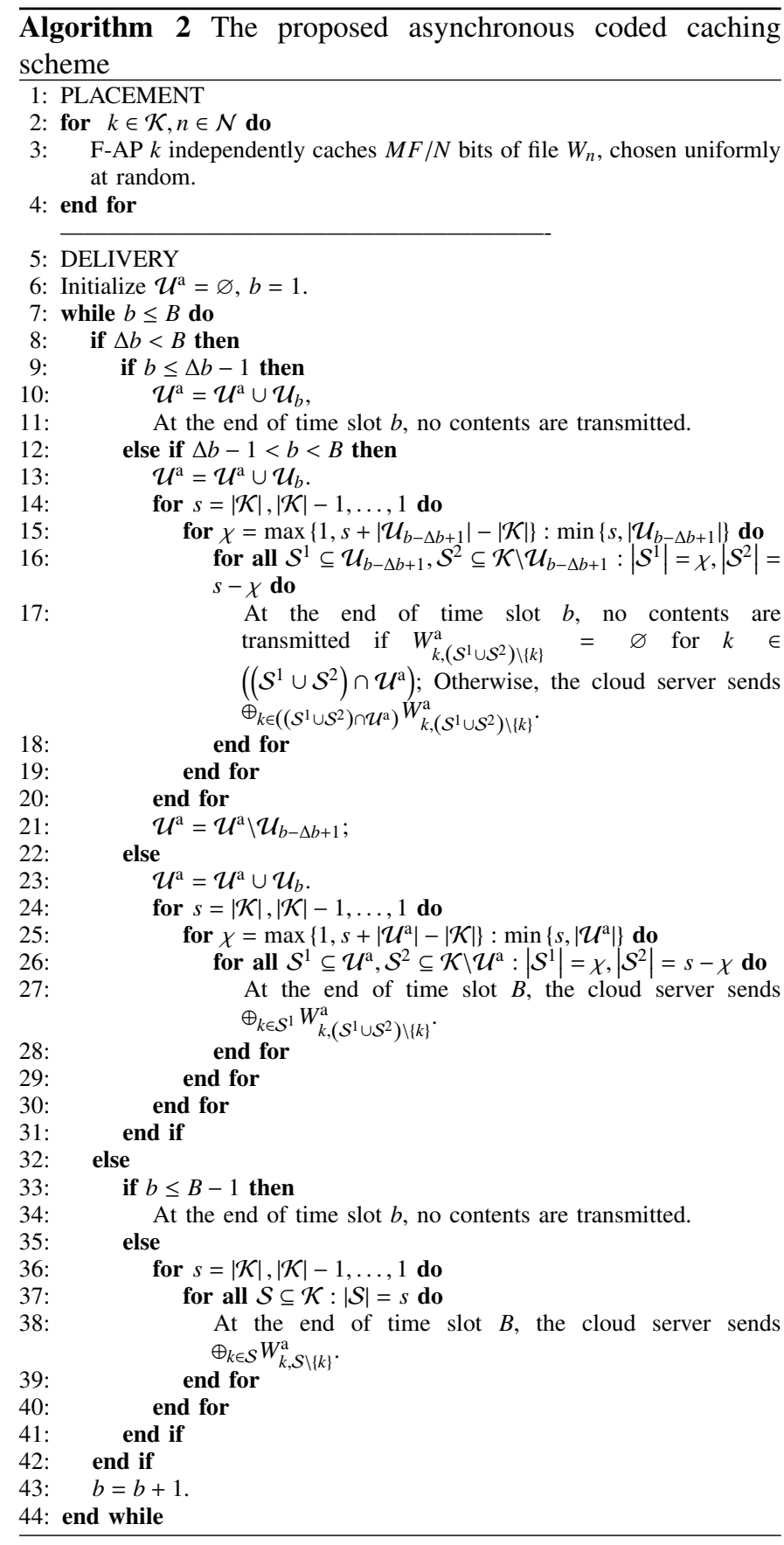

Example 3: Consider the same setting as Example 1. It can be readily seen that this corresponds to the worst-case request. According to Algorithm 2, the coded-multicasting contents transmitted by the cloud server at the end of time slot 2,3, and 4 are illustrated in Table I, Table II, and Table III, respectively. Note that $\varnothing$ indicates that no contents are transmitted in the tables. In addition, subfile $W_{3,\{2,4\}}^{\mathrm{a}}$ is actually $\varnothing$ according to the updated cache records.

Still consider the same setting as Example 1. We explain here how Algorithm 2 implements our proposed encoding set partition method. Focus on $\mathcal{S}=\{1,2,3,4\}$. Firstly, no contents are transmitted at the end of time slot 1 . At the end of time slot $2, W_{1,\{2,3,4\}}^{\mathrm{a}} \oplus W_{2,\{1,3,4\}}^{\mathrm{a}}$ is transmitted with $\mathcal{U}^{\mathrm{a}}=\{1,2\}$. At the end of time slot 3 , the cloud server decides not to
TABLE I. The contents transmitted during time slot 2

\begin{tabular}{|c|c|c|c|c|c|}
\hline$s$ & $\chi$ & $\mathcal{S}^{1}$ & $\mathcal{S}^{2}$ & $\mathcal{U}^{\mathrm{a}}$ & Coded-multicasting content \\
\hline 4 & 1 & $\{1\}$ & $\{2,3,4\}$ & $\{1,2\}$ & $W_{1,\{2,3,4\}}^{\mathrm{a}} \oplus W_{2,21,3,4\}}^{\mathrm{a}}$ \\
\hline 3 & 1 & $\{1\}$ & $\{2,3\}$ & $\{1,2\}$ & $W_{1,\{2,3\}}^{\mathrm{a}} \oplus W_{2,21,3\}}^{\mathrm{a}}$ \\
\hline 3 & 1 & $\{1\}$ & $\{2,4\}$ & $\{1,2\}$ & $W_{1,\{2,4\}}^{\mathrm{a}} \oplus W_{2,\{1,4\}}^{\mathrm{a}}, W_{1,\{3,4\}}^{\mathrm{a}}$ \\
\hline 3 & 1 & $\{1\}$ & $\{3,4\}$ & $\{1,2\}$ & $W_{1,\{2\}}^{\mathrm{a}} \oplus W_{2,\{1\}}^{\mathrm{a}}$ \\
\hline 2 & 1 & $\{1\}$ & $\{2\}$ & $\{1,2\}$ & $W_{1,\{3\}}^{\mathrm{a}}$ \\
\hline 2 & 1 & $\{1\}$ & $\{3\}$ & $\{1,2\}$ & $W_{1,\{4\}}^{\mathrm{a}}$ \\
\hline 2 & 1 & $\{1\}$ & $\{4\}$ & $\{1,2\}$ & $W_{1, \varnothing}^{\mathrm{a}}$ \\
\hline 1 & 1 & $\{1\}$ & $\varnothing$ & $\{1,2\}$ & \multicolumn{3}{c}{} \\
\hline
\end{tabular}

TABLE II. The contents transmitted during time slot 3

\begin{tabular}{|c|c|c|c|c|c|}
\hline$s$ & $\chi$ & $\mathcal{S}^{1}$ & $\mathcal{S}^{2}$ & $\mathcal{U}^{\mathrm{a}}$ & Coded-multicasting content \\
\hline 4 & 1 & $\{2\}$ & $\{1,3,4\}$ & $\{2,3\}$ & $\varnothing$ \\
\hline 3 & 1 & $\{2\}$ & $\{1,3\}$ & $\{2,3\}$ & $\varnothing$ \\
\hline 3 & 1 & $\{2\}$ & $\{1,4\}$ & $\{2,3\}$ & $\varnothing$ \\
\hline 3 & 1 & $\{2\}$ & $\{3,4\}$ & $\{2,3\}$ & $W_{2,\{3,4\}}^{\mathrm{a}} \oplus W_{3,\{2,4\}}^{\mathrm{a}}$ \\
\hline 2 & 1 & $\{2\}$ & $\{1\}$ & $\{2,3\}$ & $\varnothing$ \\
\hline 2 & 1 & $\{2\}$ & $\{3\}$ & $\{2,3\}$ & $W_{2,\{3\}}^{\mathrm{a}} \oplus W_{3,\{2\}}^{\mathrm{a}}$ \\
\hline 2 & 1 & $\{2\}$ & $\{4\}$ & $\{2,3\}$ & $W_{2,34}^{\mathrm{a}}$ \\
\hline 1 & 1 & $\{2\}$ & $\varnothing$ & $\{2,3\}$ & $W_{2, \varnothing}^{\mathrm{a}}$ \\
\hline
\end{tabular}

TABLE III. The contents transmitted during time slot 4

\begin{tabular}{|c|c|c|c|c|c|}
\hline$s$ & $\chi$ & $\mathcal{S}^{1}$ & $\mathcal{S}^{2}$ & $\mathcal{U}^{\mathrm{a}}$ & Coded-multicasting content \\
\hline 4 & 2 & $\{3,4\}$ & $\{1,2\}$ & $\{3,4\}$ & $W_{3,\{1,2,4\}}^{\mathrm{a}} \oplus W_{4,\{1,2,3\}}^{\mathrm{a}}$ \\
\hline 3 & 1 & $\{3\}$ & $\{1,2\}$ & $\{3,4\}$ & $W_{3,\{1,2\}}^{\mathrm{a}}$ \\
\hline 3 & 1 & $\{4\}$ & $\{1,2\}$ & $\{3,4\}$ & $W_{4,\{1,2\}}^{\mathrm{a}}$ \\
\hline 3 & 2 & $\{3,4\}$ & $\{1\}$ & $\{3,4\}$ & $W_{3,\{1,4\}}^{\mathrm{a}} \oplus W_{4,\{1,3\}}^{\mathrm{a}}$ \\
\hline 3 & 2 & $\{3,4\}$ & $\{2\}$ & $\{3,4\}$ & $W_{3,\{2,4\}}^{\mathrm{a}}(\varnothing) \oplus W_{4,\{2,3\}}^{\mathrm{a}}$ \\
\hline 2 & 1 & $\{3\}$ & $\{1\}$ & $\{3,4\}$ & $W_{3,\{1\}}^{\mathrm{a}}$ \\
\hline 2 & 1 & $\{3\}$ & $\{2\}$ & $\{3,4\}$ & $\varnothing$ \\
\hline 2 & 1 & $\{4\}$ & $\{1\}$ & $\{3,4\}$ & $W_{4,\{1\}}^{\mathrm{a}}$ \\
\hline 2 & 1 & $\{4\}$ & $\{2\}$ & $\{3,4\}$ & $W_{4,\{2\}}^{\mathrm{a}}$ \\
\hline 2 & 2 & $\{3,4\}$ & $\varnothing$ & $\{3,4\}$ & $W_{3,\{4\}}^{\mathrm{a}} \oplus W_{4,\{3\}}^{\mathrm{a}}, W_{3, \varnothing}^{\mathrm{a}}$ \\
\hline 1 & 1 & $\{3\}$ & $\varnothing$ & $\{3,4\}$ & $W_{4, \varnothing}^{\mathrm{a}}$ \\
\hline 1 & 1 & $\{4\}$ & $\varnothing$ & $\{3,4\}$ & \\
\hline
\end{tabular}

transmit $W_{2,\{1,3,4\}}^{\mathrm{a}} \oplus W_{3,\{1,2,4\}}^{\mathrm{a}}$ with $\mathcal{U}^{\mathrm{a}}=\{2,3\}$, since $W_{2,\{1,3,4\}}^{\mathrm{a}}$ is $\varnothing$ according to the updated cache records. Correspondingly, no contents are transmitted. Finally, $W_{3,\{1,2,4\}}^{\mathrm{a}} \oplus W_{4,\{1,2,3\}}^{\mathrm{a}}$ is transmitted with $\mathcal{U}^{\mathrm{a}}=\{3,4\}$ at the end of time slot 4 . It can be readily seen that $W_{1,\{2,3,4\}}^{\mathrm{s}} \oplus W_{2,\{1,3,4\}}^{\mathrm{s}} \oplus W_{3,\{1,2,4\}}^{\mathrm{s}} \oplus W_{4,\{1,2,3\}}^{\mathrm{s}}$ is partitioned into two parts of equal size, i.e., $W_{1,\{2,3,4\}}^{\mathrm{a}} \oplus W_{2,\{1,3,4\}}^{\mathrm{a}}$ and $W_{3,\{1,2,4\}}^{\mathrm{a}} \oplus W_{4,\{1,2,3\}}^{\mathrm{a}}$, for transmission in our proposed asynchronous coded caching scheme.

Remark 3: The major innovation of our proposed scheme is to partition the coded-multicasting contents in synchronous coded caching by using our proposed encoding set partition method. Our contributions are mainly reflected in selecting a part of the file library for further processing based on the request arrival status of the considered time slot. Our proposed scheme can create considerable coded-multicasting opportunities while the maximum request delay of each F-AP is no more than $\Delta b$ time slots.

Remark 4: Both Maddah-Ali-Niesen's decentralized scheme in [8] and our proposed scheme are time-slot based ones. However, in Maddah-Ali-Niesen's asynchronous coded 
caching scheme, only simple extension from synchronous coded caching is considered. Just as shown in the provided example, during the first and last two time slots, no coded multicasting opportunities have been created. In comparison, in our proposed scheme, we try to create sufficient coded multicasting opportunities under the constraint of the maximum request delay $\Delta b$. Specifically, when $\Delta b>1$, the cloud server does not transmit any content at the end of time slot $1,2, \ldots, \Delta b-1$ in order that more coded multicasting opportunities can be created in the subsequent time slots. Besides, at the end of the last time slot $B$, coded multicasting opportunities have been created to fulfil all the requests of the active F-APs.

Remark 5: In [29], the asynchronous coded caching problem has been considered mainly for the offline case, where the authors presented their proposed approach based on a system using the centralized synchronous coded caching scheme in [7]. Furthermore, the authors proposed a linear programming formulation that minimizes the overall rate from the server subject to the constraint that each user meets its deadline. In comparison, we propose a decentralized asynchronous coded caching scheme based on a different system model for the online case, which is more applicable for practical scenarios, and can work well for both the online case and offline case. Moreover, we propose an encoding set collapsing rule and an encoding set partition method to minimize the worst-case normalized fronthual load subject to a given maximum request delay.

Remark 6: In the delivery phase, the cloud server needs to partition $N$ files according to the updated cache records at the end of time slot $\Delta b, \Delta b+1, \ldots, B$. In practice, the cloud server only needs to partition $N$ files based on the initial cache records before starting transmission, and updates the sizes of the subfiles in the cache records that are encoded to be transmitted by setting them to $\varnothing$ directly after completing transmission at the end of each time slot.

\section{Performance Analysis of the Proposed ASYNCHRONOUS CODED CACHING SCHEME}

To emphasize the dependence of the fronthaul load on the cache size $M$, the number of files $N$, the number of FAPs $K$ and the maximum request delay, let $R_{A}(M, N, K, \Delta b)$ denote the fronthaul load of our proposed asynchronous coded caching scheme. As the request distribution during the $B$ time slots affects $R_{A}(M, N, K, \Delta b)$, it is hard to obtain its exact expression. Focusing on the special case with $\left|\mathcal{U}_{b}\right|=L$, we can derive the closed-form expression of $R_{A}(M, N, K, \Delta b)$. As for the general case with random $\left|\mathcal{U}_{b}\right|$, we can establish the upper and lower bounds of $R_{A}(M, N, K, \Delta b)$.

\section{A. Special Case with $\left|\mathcal{U}_{b}\right|=L$}

Let $\left|W_{k, \mathcal{S} \backslash\{k\}}^{\mathrm{a}}\right|$ denote the size of $W_{k, \mathcal{S} \backslash\{k\}}^{\mathrm{a}}$. According to the law of large numbers, we have

$$
\left|W_{k, \mathcal{S} \backslash\{k\}}^{\mathrm{a}}\right| \approx(M / N)^{s-1}(1-M / N)^{K-(s-1)} F .
$$

Theorem 1: Consider the special case with $\left|\mathcal{U}_{b}\right|=L$ and $B \geq 3$. For large enough $F$, the fronthaul load is arbitrarily close to

$$
R_{A}(M, N, K, \Delta b)=\sum_{s=1}^{K}\left|W_{k, \mathcal{S} \backslash\{k\}}^{\mathrm{a}}\right| \sum_{Y=\left\lceil\frac{s}{\Delta b \cdot L}\right\rceil}^{\min \left\{\left\lceil\frac{B}{\Delta b}\right\rceil, s\right\}} q(s, Y, \Delta b) Y,
$$

where $q(s, Y, \Delta b)$ is shown at the bottom of this page with

$$
\begin{aligned}
& q_{1}\left(s, Y, \Delta b^{\prime}, \Delta b\right)\left(\begin{array}{c}
B-\Delta b^{\prime}+(Y-1)(\Delta b-1) \\
Y-1
\end{array}\right) \\
& \quad \times \sum_{\alpha=\max \left\{Y, s-\left((Y-1) \Delta b+\Delta b^{\prime}-Y\right) L\right\}}^{\min \{s, Y L\}} b(Y, \alpha)
\end{aligned}\left(\begin{array}{c}
\left((Y-1) \Delta b+\Delta b^{\prime}-Y\right) L \\
s-\alpha
\end{array}\right),
$$

$$
\begin{aligned}
& q_{2}(s, Y, \Delta b) \\
& =\left\{\begin{array}{cc}
\left(\begin{array}{c}
B \\
Y
\end{array}\right) b(Y, s), & \Delta b=1, \\
\left(\begin{array}{c}
B-Y(\Delta b-1) \\
Y
\end{array}\right) & 1<\Delta b<B, \\
\times \sum_{\alpha=\max \{Y, s-Y(\Delta b-1) L\}}^{\min \{s, Y L\}} b(Y, \alpha)\left(\begin{array}{c}
Y(\Delta b-1) L \\
s-\alpha
\end{array}\right), &
\end{array}\right.
\end{aligned}
$$$$
b(Y, \alpha)= \begin{cases}\left(\begin{array}{c}
L \\
\alpha
\end{array}\right), & Y=1,1 \leq \alpha \leq L \\
\left(\begin{array}{c}
L \\
1
\end{array}\right), & Y>1, \alpha=Y \\
1, & Y>1, L>1, \alpha=Y L \\
\min \{L, \alpha-(Y-1)\}_{\sum_{v=1}^{Y}\left(\begin{array}{c}
L \\
v
\end{array}\right) b(Y-1, \alpha-v),} & \text { else. }\end{cases}
$$

Proof: Please see appendix A.

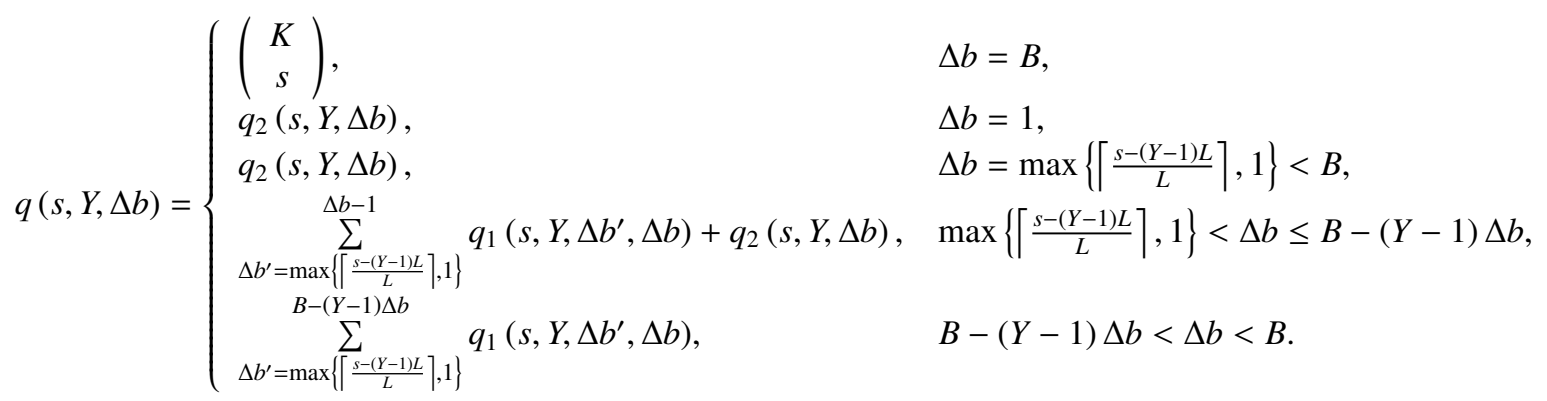


Remark 6: when $B=2$, we have

$$
q(s, Y, \Delta b)=\left\{\begin{array}{cc}
\left(\begin{array}{c}
K \\
s
\end{array}\right), & \Delta b=2, \\
q_{2}(s, Y, \Delta b), & \Delta b=1 .
\end{array}\right.
$$

For description convenience, the case where $B=2$ is omitted in Theorem 1.

Let $R_{S}(M, N, K)$ denote the fronthaul load of the MaddahAli-Niesen's decentralized synchronous coded caching scheme. From [8], we have

$$
\begin{aligned}
R_{S}(M, N, K) & =F \sum_{s=1}^{K}\left(\begin{array}{c}
K \\
s
\end{array}\right)(M / N)^{s-1}(1-M / N)^{K-(s-1)} \\
& =F K(1-M / N) \frac{N}{K M}\left(1-(1-M / N)^{K}\right), \quad N \geq K .
\end{aligned}
$$

According to Theorem 1, we also have

$$
\begin{aligned}
\left.R_{A}(M, N, K, \Delta b)\right|_{\Delta b=B} & =F \sum_{s=1}^{K}\left|W_{k, \mathcal{S} \backslash\{k\} \mid}^{\mathrm{a}}\right| \sum_{Y=\left\lceil\frac{s}{B L}\right\rceil}^{\min \{1, s\}} q(s, Y, \Delta b) Y \\
& =F \sum_{s=1}^{K}\left|W_{k, \mathcal{a} \backslash\{k\} \mid}^{\mathrm{a}}\right| \sum_{Y=1}^{1} q(s, Y, \Delta b) Y \\
& =F \sum_{s=1}^{K}(M / N)^{s-1}(1-M / N)^{K-(s-1)}\left(\begin{array}{c}
K \\
s
\end{array}\right) \\
& =R_{S}(M, N, K), \quad N \geq K .
\end{aligned}
$$

It can be readily seen that our proposed scheme has the same fronthaul load as the Maddah-Ali-Niesen's decentralized scheme when $\Delta b=B$.

\section{B. General Case with Random $\left|\mathcal{U}_{b}\right|$}

Theorem 2: The fronthaul load of our proposed scheme is bounded as follows

$$
\begin{aligned}
R_{S}(M, N, K) & \leq R_{A}(M, N, K, \Delta b) \\
& \leq F K\left(1-\frac{M}{N}\right) \min \left\{\left\lceil\frac{B}{\Delta b}\right\rceil \frac{N}{K M}\left(1-(1-M / N)^{K}\right), 1\right\} .
\end{aligned}
$$

Proof: Please see appendix B.

It can be readily seen that the lower bound of the fronthual load of our proposed scheme is the same as that of the Maddah-Ali-Niesen's decentralized synchronous coded caching scheme, and also does not consider the asynchronous case, which is of great challenge. According to (15), our proposed scheme has the same fronthaul load as the MaddahAli-Niesen's decentralized scheme when $\Delta b=B$ and $\left|\mathcal{U}_{b}\right|=L$. It can be seen that the lower bound can indeed be achieved for the above special case. However, as can be seen from (8)-(12), even for the special case with $\left|\mathcal{U}_{b}\right|=L$, the expression of the fronthual load of our proposed scheme, i.e., $R_{A}(M, N, K, \Delta b)$, is already extremely complicated. Therefore, we can readily see that it will be very difficult to obtain the expression or the lower bound (considering the asynchronous case) of $R_{A}(M, N, K, \Delta b)$ for the general case with random $\left|\mathcal{U}_{b}\right|$.

Comparing the fronthaul load of our proposed scheme with that of the Maddah-Ali-Niesen's decentralized scheme, we

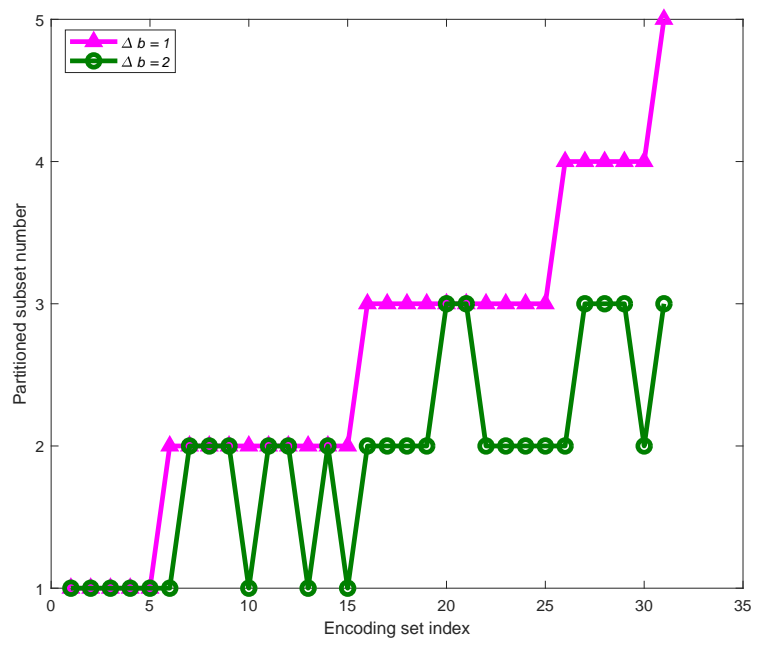

Fig. 2. The number of encoding subsets such that each encoding set can be partitioned into with $B=5$ and $\mathcal{U}_{b}=\{b\}$.

have the following corollary.

Corollary 1:

$$
1 \leq \frac{R_{A}(M, N, K, \Delta b)}{R_{S}(M, N, K)} \leq\lceil B / \Delta b\rceil, \quad N \geq K .
$$

As for the general case of asynchronous requests, Theorem 2 and Corollary 1 show that the fronthaul load of our proposed scheme is at most a factor $\lceil B / \Delta b\rceil$ larger than that of the Maddah-Ali-Niesen's decentralized scheme. When $\Delta b<B$, the performance gap between our proposed scheme and the Maddah-Ali-Niesen's decentralized scheme is due to the fact that asynchronous requests lead to the loss of codedmulticasting opportunities, which is also the key difference between asynchronous and synchronous coded caching.

According to (5), the number of partitioned encoding subsets for an encoding set with a smaller $\Delta b$ is relatively larger, which can be illustrated in Fig. 2. Note that $R_{A}(M, N, K, \Delta b)$ can be calculated by accumulating the sizes of the codedmulticasting contents corresponding to the subsets that al1 the encoding sets are partitioned into. Correspondingly, $R_{A}(M, N, K, \Delta b)$ increases with $\Delta b$, since the size of the codedmulticasting content transmitted for an encoding set by the cloud server is unchanged. Moreover, for $M \in[N / K, N]$, the fronthaul load of the Maddah-Ali-Niesen's decentralized scheme can be up to a factor $K$ smaller than that of the uncoded caching scheme [8]. Besides, when the number of F-APs increases for $M \in[N / K, N]$, the maximum request delay can be set to a relatively smaller value with the same considerable coded-multicasting opportunities created.

\section{Simulation Results}

In this section, the performance of our proposed decentralized asynchronous coded caching scheme is evaluated via simulations. We adopt the Maddah-Ali-Niesen's decentralized scheme and the uncoded caching scheme as baselines. The system parameters are set as follows: $F=1 \mathrm{~Gb}, N=100$, $T=10 \mathrm{~s}, B=5$. 


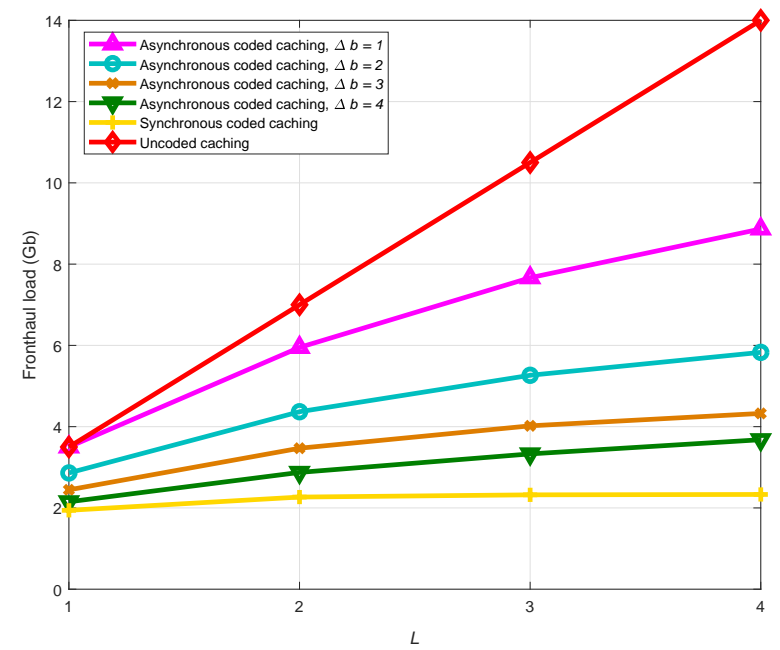

Fig. 3. Fronthaul load versus $L$ with $M=30$.

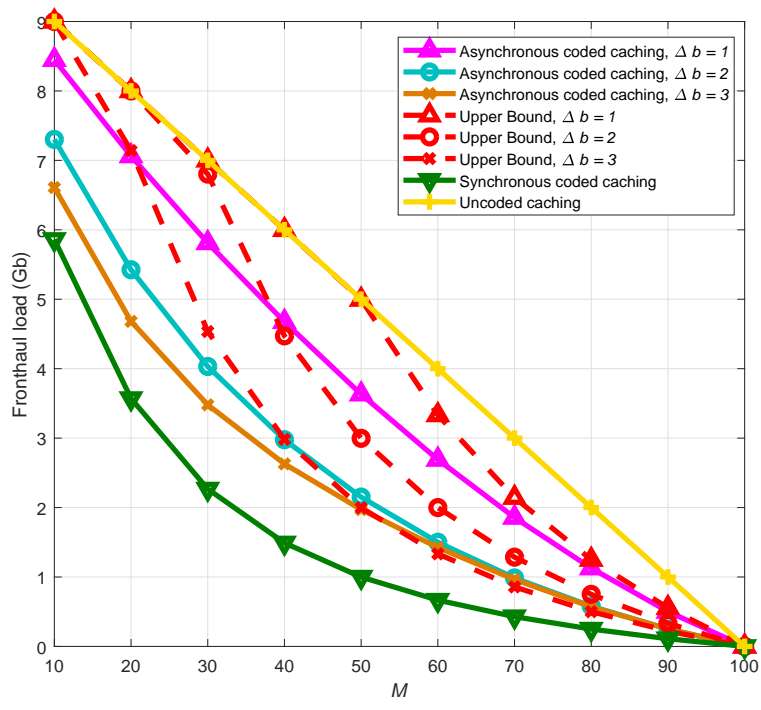

Fig. 4. Fronthaul load versus $M$ with $K=10$.

In Fig. 3, we show the effect of the number of F-APs requesting contents during each time slot on the fronthaul load of each scheme with $M=30$ for different $\Delta b$. As the request distribution affects the fronthaul load, we consider the special case with $\left|\mathcal{U}_{b}\right|=L$. As shown, the fronthaul load of our proposed scheme increases more slowly with $L$ compared with that of the uncoded caching scheme. The reason for this result is that more coded-multicasting opportunities can be created when $L$ increases, i.e., $K$ increases. Correspondingly, when $K$ increases for $M \in[N / K, N]$, the maximum request delay can be set to a relatively smaller value while the same considerable coded-multicasting opportunities are created.

In Fig. 4, we show the effect of the normalized cache size of each F-AP, i.e., $M$, on the fronthaul load of each scheme with $K=10$ for different $\Delta b$. Here we consider the general case with random $\left|\mathcal{U}_{b}\right|$. As shown, our proposed scheme can cre-

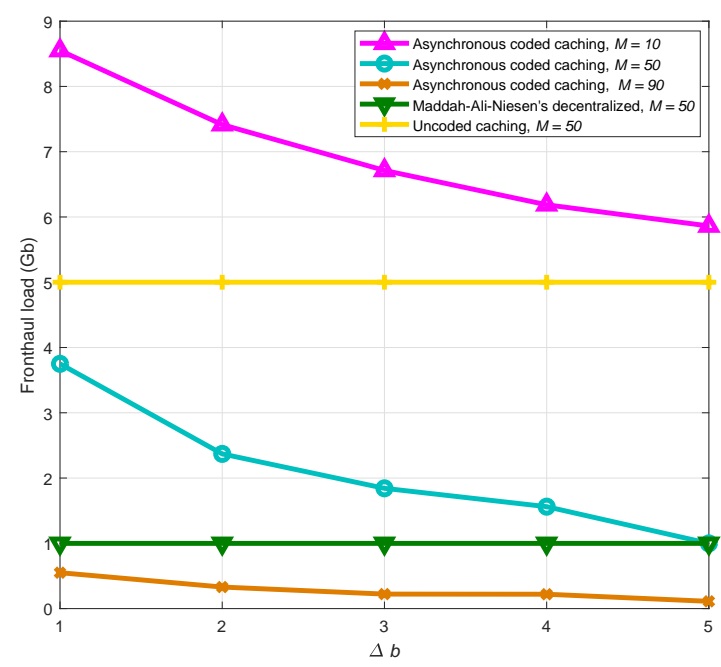

Fig. 5. Fronthaul load versus $\Delta b$ for varying cache sizes with $K=10$.

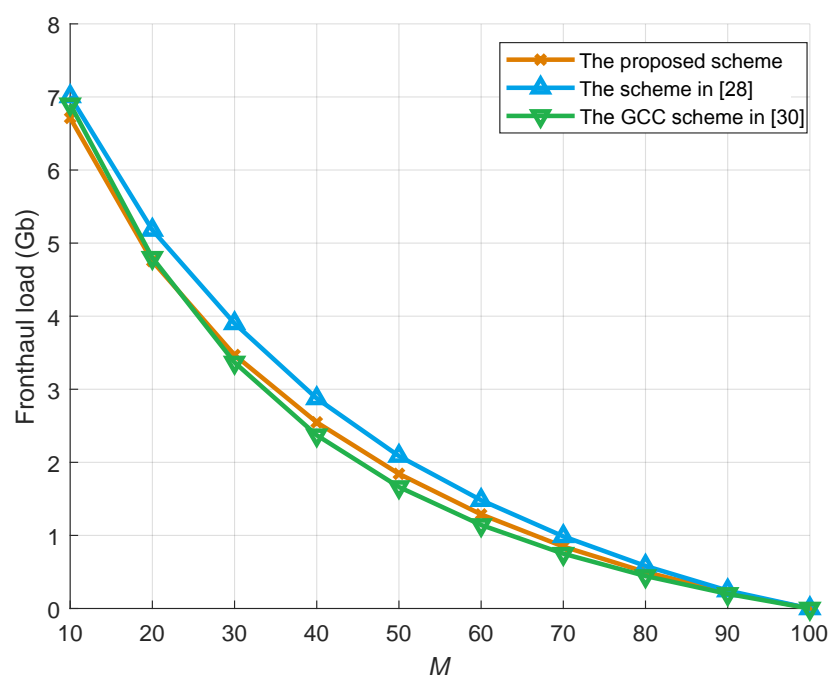

Fig. 6. Fronthaul load versus $M$ for different asynchronous coded caching schemes.

ate considerable coded-multicasting opportunities compared with the uncoded caching scheme. Moreover, the fronthaul load decreases and its slope increases when $M$ increases, which is the same as the Maddah-Ali-Niesen's decentralized scheme. Furthermore, the fronthaul load of our proposed scheme is between the lower bound, i.e., the fronthaul load of the Maddah-Ali-Niesen's decentralized synchronous coded caching scheme, and the upper bound, and approaches the upper bound when $M$ increases.

In Fig. 5, we show how $\Delta b$ affects the fronthaul load of each scheme for varying cache sizes with random $\left|\mathcal{U}_{b}\right|$ and $K=10$. As shown, the fronthaul load of our proposed scheme decreases with $\Delta b$, which means that our proposed scheme can create more coded-multicasting opportunities with a relaxed 
delay requirement. Furthermore, the larger $\Delta b$ is, the more the decrease of the fronthaul load of our proposed scheme is in comparison with that of the uncoded caching scheme. The performance gap between the fronthaul load of our proposed scheme and that of the Maddah-Ali-Niesen's decentralized scheme is smaller when $\Delta b$ is larger. The reason for the above results is that a larger $\Delta b$ leads to a smaller number of the partitioned subsets. Besides, as $\Delta b$ determines the maximum request delay, it can be set to a relatively small value in delaysensitive scenarios and adjusted flexibly to achieve the loaddelay tradeoff in other scenarios.

In Fig. 6, we show the performance comparison among our proposed scheme, the scheme in [28], and the GCC scheme in [30]. In the simulations, the maximum request delay $\Delta b$ is set to 3 for our proposed scheme and the threshold $\tau$ is set to 3 for the scheme in [28]. As shown, the performance of our proposed scheme is better than that in [28]. The reason is that the scheme in [28] puts all requests into a sequence, and a new request can be merged with the queued requests but may miss to merge with the upcoming requests for the $\tau$-fit threshold rule, which is more appropriate to the situations where the sequence of requests is short, the file size is small, or the number of users is not large. Although the performance gap between our proposed scheme and the scheme in [28] with $\tau=3$ is small, it is not always the case. The performance gap will become larger when the maximum request delay $\Delta b$, the threshold $\tau$, the queue length or the F-AP number is larger. According to [28], it can be readily verified that our proposed scheme satisfies the perfect-fit rule with $\tau=0$, i.e., 0 -fit threshold rule. Moreover, a larger $\tau$ or queue length can indeed bring larger performance gap. Besides, with a larger $\Delta b$ or F-AP number, the coded-multicasting opportunities among the considered F-APs will increase. Correspondingly, the scheme in [28] will result in a larger probability to miss the coded-multicasting opportunities, and the performance gap will become larger. Furthermore, the GCC scheme in [30] has a slight better performance than our proposed scheme when $M \geq 20$. The reason is that the GCC scheme is centralized and the server knows the identity and the number of users exactly in the placement phase. Correspondingly, it can be carefully designed to create more coded-multicasting opportunities. However, the GCC scheme in [30] cannot be applied to the networks with a variable number of users whereas both our proposed scheme and the scheme in [28] can be due to their decentralized property. As for the complexity, in the delivery phase, the GCC scheme in [30] and our proposed scheme need some loops to encode the subfiles in each time slot, and the number of loops in a time slot can be calculated to be $\left\{\begin{array}{cc}O\left(\left(\begin{array}{c}K \\ \frac{K M}{N}+1\end{array}\right)-\left(\begin{array}{c}K-\Delta b L \\ \frac{K M}{N}+1\end{array}\right)\right), & \Delta b L \leq K\left(1-\frac{M}{N}\right)-1, \\ O\left(\left(\begin{array}{c}K \\ N\end{array}\right)\right), & \text { else. }\end{array}\right.$ and $O\left(2^{K}-\right.$ traverse the sequence of requests multiple times, and the maximum number of traversals in a time slot can be calculated to be $O\left(1 / 2 L F^{\prime}(1-M / N)\left[2 L F^{\prime}(1-M / N) \Delta b+(L-1)\right]\right)$, where $F^{\prime}$ denotes the number of partitioned subfiles for each file.

\section{CONCLUSIONS}

In this paper, we have proposed a decentralized asynchronous coded caching scheme for the online case in FRANs where users asynchronously request contents with the maximum request delay. Our proposed scheme provides asynchronous and synchronous transmission methods to fulfill the delay requirements of different practical scenarios. The analytical results have shown that the fronthaul load of our proposed scheme is at most a constant factor larger than that of the Maddah-Ali- Niesen's decentralized scheme for a given maximum request delay. The simulation results have shown that more coded-multicasting opportunities can be created when the maximum request delay increases in asynchronous request scenarios. For the future work, we would like to explore asynchronous coded caching with a nonuniform popularity distribution.

\section{REFERENCES}

[1] G. Lee, W. Saad, and M. Bennis, "An online secretary framework for fog network formation with minimal latency," in 2017 IEEE Int. Conf. Commun. (ICC), May 2017, pp. 1-6.

[2] M. S. ElBamby, M. Bennis, and W. Saad, "Proactive edge computing in latency-constrained fog networks," in the 26th European Conf. Netw. Commun. (EuCnC), June 2017, pp. 1-6.

[3] K. Zhang, Y. Mao, S. Leng, Q. Zhao, L. Li, X. Peng, L. Pan, S. Maharjan, and Y. Zhang, "Energy-efficient offloading for mobile edge computing in 5G heterogeneous networks," IEEE Access, vol. 4, pp. 5896-5907, Aug. 2016.

[4] E. Bastug, M. Bennis, and M. Debbah, "Living on the edge: The role of proactive caching in 5G wireless networks," IEEE Commun. Mag., vol. 52, no. 8, pp. 82-89, Aug. 2014.

[5] X. Wang, M. Chen, T. Taleb, A. Ksentini, and V. C. M. Leung, "Cache in the air: Exploiting content caching and delivery techniques for 5G systems," IEEE Commun. Mag., vol. 52, no. 2, pp. 131-139, Feb. 2014.

[6] Y. Jiang, M. Ma, M. Bennis, F. Zheng, and X. You, "User preference learning based edge caching for fog radio access network," IEEE Trans. Commun. (Early Access), pp. 1-16, Nov. 2018.

[7] M. A. Maddah-Ali and U. Niesen, "Fundamental limits of caching," IEEE Trans. Inf. Theory, vol. 60, no. 5, pp. 2856-2867, May 2014

[8] — , "Decentralized coded caching attains order-optimal memory-rate tradeoff," IEEE/ACM Trans. Netw., vol. 23, no. 4, pp. 1029-1040, Aug. 2015.

[9] K. Shanmugam, M. Ji, A. M. Tulino, J. Llorca, and A. G. Dimakis., "Finite-length analysis of caching-aided coded multicasting," IEEE Trans. Inf. Theory, vol. 62, no. 10, pp. 5524-5537, Oct. 2016.

[10] S. Jin, Y. Cui, H. Liu, and G. Caire, "Order-optimal decentralized coded caching schemes with good performance in finite file size regime," in 2016 IEEE Global Commun. Conf. (GLOBECOM), Dec. 2016, pp. 1-7.

[11] U. Niesen and M. A. Maddah-Ali, "Coded caching with nonuniform demands," IEEE Trans. Inf. Theory, vol. 63, no. 2, pp. 1146-1158, Feb. 2017.

[12] J. Hachem, N. Karamchandani, and S. Diggavi, "Multi-level coded caching," in 2014 IEEE Int. Symp. Inf. Theory, June 2014, pp. 56-60.

[13] M. Ji, A. M. Tulino, J. Llorca, and G. Caire, "Order-optimal rate of caching and coded multicasting with random demands," IEEE Trans. Inf. Theory, vol. 63, no. 6, pp. 3923-3949, June 2017.

[14] J. Zhang, X. Lin, and X. Wang, "Coded caching under arbitrary popularity distributions," IEEE Trans. Inf. Theory, vol. 64, no. 1, pp. 349-366, Jan. 2018.

[15] M. Ji, A. M. Tulino, J. Llorca, and G. Caire, "Caching and coded multicasting: Multiple groupcast index coding," in 2014 IEEE Global Conf. Signal Inf. Process. (GlobalSIP), Dec. 2014, pp. 881-885.

[16] M. Ji, K. Shanmugam, G. Vettigli, J. Llorca, A. M. Tulino, and G. Caire, "An efficient multiple-groupcast coded multicasting scheme for finite fractional caching," in 2015 IEEE Int. Conf. Commun. (ICC), June 2015, pp. 3801-3806.

[17] J. Zhang, X. Lin, C. C. Wang, and X. Wang, "Coded caching for files with distinct file sizes," in 2015 IEEE Int. Symp. Inf. Theory (ISIT), June 2015, pp. 1686-1690. 
[18] M. M. Amiri, Q. Yang, and D. Gndz, "Decentralized caching and coded delivery with distinct cache capacities," IEEE Trans. Commun., vol. 65, no. 11, pp. 4657-4669, Nov. 2017.

[19] R. Pedarsani, M. A. Maddah-Ali, and U. Niesen, "Online coded caching," IEEE/ACM Trans. Netw., vol. 24, no. 2, pp. 836-845, Apr. 2016.

[20] Q. Yan, M. Cheng, X. Tang, and Q. Chen, "On the placement delivery array design for centralized coded caching scheme," IEEE Trans. Inf. Theory, vol. 63, no. 5, pp. 5821-5833, Sep. 2017.

[21] C. Shangguan, Y. Zhang, and G. Ge, "Centralized coded caching schemes: A hypergraph theoretical approach," IEEE Trans. Inf. Theory, vol. 64 , no. 8, pp. 5755-5766, Aug. 2018.

[22] L. Tang and A. Ramamoorthy, "Coded caching schemes with reduced subpacketization from linear block codes," IEEE Trans. Inf. Theory, vol. 64, no. 4, pp. 3099-3120, Apr. 2018.

[23] H. Ghasemi and A. Ramamoorthy, "Improved lower bounds for coded caching," IEEE Trans. Inf. Theory, vol. 63, no. 7, pp. 4388-4413, Jul. 2017.

[24] C. Tian and J. Chen, "Caching and delivery via interference elimination," IEEE Trans. Inf. Theory, vol. 64, no. 3, pp. 1548-1560, Mar. 2018.

[25] J. Gómez-Vilardebó, "A novel centralized coded caching scheme with coded prefetching," IEEE J. Sel. Areas Commun., vol. 36, no. 6, pp. 1165-1175, Jun. 2018.

[26] K. Zhang and C. Tian, "Fundamental limits of coded caching: From uncoded prefetching to coded prefetching," IEEE J. Sel. Areas Commun., vol. 63, no. 6, pp. 1153-1164, Jun. 2018.

[27] M. A. Maddah-Ali and U. Niesen, "Coding for caching: Fundamental limits and practical challenges," IEEE Commun. Mag., vol. 54, no. 8, pp. 23-29, Aug. 2016.

[28] U. Niesen and M. A. Maddah-Ali, "Coded caching for delay-sensitive content," in 2015 IEEE Int. Conf. Commun. (ICC), June 2015, pp. 55595564.

[29] H. Ghasemi and A. Ramamoorthy, "Asynchronous coded caching," in 2017 IEEE Int. Symp. Inf. Theory (ISIT), June 2017, pp. 2438-2442.

[30] Y. Lu, W. Chen, and H. V. Poor, "Coded joint pushing and caching with asynchronous user requests," IEEE J. Sel. Areas Commun., vol. 36, no. 8, pp. 1843-1856, Aug. 2018.

[31] W. Huang, Y. Jiang, M. Bennis, F. Zheng, H. Gacanin, and X. You, "Decentralized asynchronous coded caching in fog-ran," in Proc. IEEE VTC 2018 Fall, Chicago, USA, Aug. 2018, pp. 1-6.

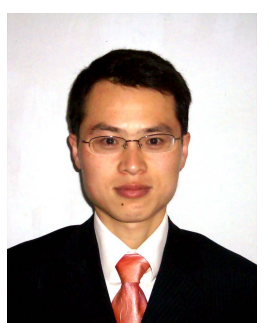

Yanxiang Jiang (S'03-M'07-SM'18) received the B.S. degree in electrical engineering from Nanjing University, Nanjing, China, in 1999 and the M.S. and $\mathrm{Ph} . \mathrm{D}$. degrees in communications and information systems from Southeast University, Nanjing, China, in 2003 and 2007, respectively.

Dr. Jiang was a Visiting Scholar with the Signals and Information Group, Department of Electrical and Computer Engineering, University of Maryland at College Park, College Park, MD, USA, in 2014. $\mathrm{He}$ is currently an Associate Professor with the National Mobile Communications Research Laboratory, Southeast University, Nanjing, China. His research interests are in the area of broadband wireless mobile communications, covering topics such as edge caching, radio resource allocation and management, fog radio access networks, small cells and heterogeneous networks, cooperative communications, green communications, device to device communications, massive MIMO, and machine learning for wireless communications.

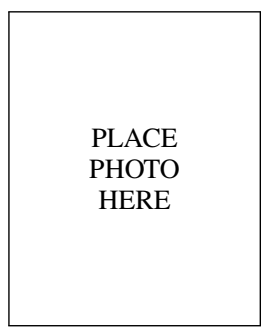

Wenlong Huang is currently pursuing the M.S. degree in communications and information systems from Southeast University, Nanjing, China.

His research interests include radio resource management and edge caching.

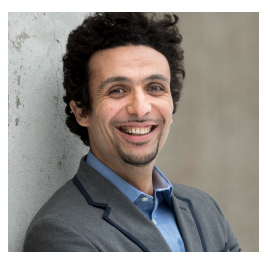

Mehdi Bennis (S'07-AM'08-SM'15) received his M.Sc. degree in electrical engineering jointly from EPFL, Switzerland, and the Eurecom Institute, France, in 2002. He obtained his Ph.D. from the University of Oulu in December 2009 on spectrum sharing for future mobile cellular systems. Currently he is an associate professor at the University of Oulu and an Academy of Finland research fellow. His main research interests are in radio resource management, heterogeneous networks, game theory, and machine learning in 5G networks and beyond. He has co-authored one book and published more than 200 research papers in international conferences, journals, and book chapters. He was the recipient of the prestigious 2015 Fred W. Ellersick Prize from the IEEE Communications Society, the 2016 Best Tutorial Prize from the IEEE Communications Society, the 2017 EURASIP Best Paper Award for the Journal of Wireless Communications and Networks, and the 2017 all-University of Oulu Award for Research.

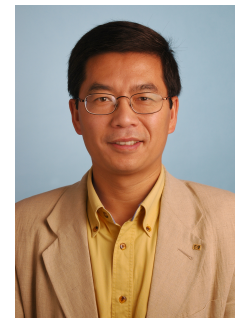

Fu-Chun Zheng (M'95-SM'99) obtained the BEng (1985) and MEng (1988) degrees in radio engineering from Harbin Institute of Technology, China, and the $\mathrm{PhD}$ degree in Electrical Engineering from the University of Edinburgh, UK, in 1992.

From 1992 to 1995 , he was a post-doctoral research associate with the University of Bradford, UK, Between May 1995 and August 2007, he was with Victoria University, Melbourne, Australia, first as a lecturer and then as an associate professor in mobile communications. He was with the University of Reading, UK, from September 2007 to July 2016 as a Professor (Chair) of Signal Processing. He has also been a distinguished adjunct professor with Southeast University, China, since 2010. Since August 2016, he has been a distinguished professor with Harbin Institute of Technology (Shenzhen), China and the University of York, UK. He has been awarded two UK EPSRC Visiting Fellowships - both hosted by the University of York (UK): first in August 2002 and then again in August 2006. Over the past two decades, Dr Zheng has also carried out many government and industry sponsored research projects - in Australia, the UK, and China. He has been both a short term visiting fellow and a long term visiting research fellow with British Telecom, UK. Dr Zheng's current research interests include signal processing for communications, multiple antenna systems, green communications, and ultra-dense networks.

He has been an active IEEE member since 1995. He was an editor (2001 - 2004) of IEEE Transactions on Wireless Communications. In 2006, Dr Zheng served as the general chair of IEEE VTC 2006-S, Melbourne, Australia (www.ieeevtc.org/vtc2006spring) - the first ever VTC held in the southern hemisphere in VTC's history of six decades. More recently he was the executive TPC Chair for VTC 2016-S, Nanjing, China (the first ever VTC held in mainland China: www.ieevtc.org/vtc2016spring). 